\title{
Estudios sobre la reactividad de harinas crudas de cemento tratadas con rápidos y diferentes calentamientos
}

\author{
Untersuchungen zur reaktivität \\ von zementrohmehlen nach verschieden \\ schneller aufheizung
}

\section{RESUMEN}

Estudios experimentales llevados a cabo a una temperatura dada de calcinación, sirvieron para observar el efecto de las altas velocidades de calentamiento sobre las reacciones del clínker.

Mientras que un aumento de la reactividad de los materiales fue claramente detectada cuando la velocidad pasó de $20 \mathrm{~K} / \mathrm{min}$ a tomar valores iguales y mayores a $100 \mathrm{~K} / \mathrm{min}$ un negativo efecto sobre el Tiempo de Reacción Completa o "Periodo de Calcinación a Punto" no pudo ser establecido. Considerando además que el tiempo necesario para llevar a la materia prima hasta la temperatura de reacción, es necesariamente un tiempo inefectivo, podrian las plantas productoras de cemento obtener mayores rendimientos en tiempo y espacio utilizando altas cuotas de calentamiento.

Estudios de microscopía probaron que con el aumento de las velocidades de calentamiento, hubo una mayor generación de microcristales incidiendo directamente sobre la molienda y calidad del clínker.

\section{ZUSAMMENFASSUNG}

Modellversuche mit linearen Aufheizgeschwindigkeiten solten zeigen, ob bei einer vorgegebenen Brenntemperatur die Verklinkerungsreaktionen beeinflußt werden können.

Während eindeutige Steigerungen der Kalkbindungsraten lediglich zwischen $20 \mathrm{~K} / \mathrm{min}$ und

Aufheizgeschwindigkeiten $\geqq 100 \mathrm{~K} /$ min nachweisbar waren, konnten negative Auswirkungen auf die

Garbranddauern bei keiner Versuchsserie festgestellt werden. Unter Berücksichtigung der zur Aufheizung der Rohstoffe notwendigen Totzeiten, können bei den technischen Zementproduktionsanlagen nach schneller Aufheizung höhere Raum-Zeit-Ausbeuten erreicht werden.

Mikroskopische Untersuchungen ergaben, da $\beta$ durch Erhöhung der Autheizraten der Anteil mikrokristalliner Klinkerphasen zunimmt, was sich direkt aut die Zementklinkermahlbarkeit und die Klinkerqualität auswirkt.

\section{INTRODUCCION Y PROBLEMATICA}

\section{I.1 Introducción}

El cemento es un aglomerante hidráulico que después de mezclarlo con agua endurece, e independientemente en el aire o bajo el agua permanece firme.

Dentro de las diferentes clases de cementos, el cemento Portland es el que posee la mayor

\section{EINLEITUNG UND PROBLEMSTELLUNG}

\section{I.1. Einführung}

Zement ist ein hydraulisches Bindemittel, das nach dem Anmachen mit Wasser selbstständig erhärtet und an der Luft und unter Wasser fest bleibt.

Unter den verschiedenen Zementarten hat der Portland-Zement die größte Bedeutung.

${ }^{\star}{ }^{\star}$ Institut für Chemische Technik der Universität Karlsruhe (TH), Bendesrepublik Deutschland.

$\left(^{+}\right)$Facultad de Ciencias Exactas, Químicas y Naturales de la Universidad Nacional de Misiones, Argentina.

$\left(^{++}\right)$Department of Chemistry in the Faculty of Mathematics and Sciences, University of Indonesia, Indonesia. 
significación. El clinker del cemento Portland, cuya fracción principal, de aproximadamente un $95 \%$ en peso representa cemento, es obtenido a partir de una mezcla de materias primas constituída principalmente por óxido de calcio $(\mathrm{CaO})$, óxido de silicio $\left(\mathrm{SiO}_{2}\right)$, óxido de aluminio $\left(\mathrm{Al}_{2} \mathrm{O}_{3}\right)$ y óxido férrico $\left(\mathrm{Fe}_{2} \mathrm{O}_{3}\right)$.

A través del calentamiento de esa mezcla hasta su sinterización, se realiza la constitución de nuevos enlaces dando lugar a la formación de las Ilamadas Fases del Clinker:

- Silicato tricálcico o Alita, $3 \mathrm{CaO} \mathrm{SiO}_{2}$, abreviado: $\mathrm{C}_{3} \mathrm{~S}$

- Silicato dicálcico o Belita, $2 \mathrm{CaO} \mathrm{SiO}_{2}$, abreviado: $\mathrm{C}_{2} \mathrm{~S}$

- Aluminato tricálcico o Aluminato, $3 \mathrm{CaO} \cdot \mathrm{Al}_{2} \mathrm{O}_{3}$, abreviado: $\mathrm{C}_{3} \mathrm{~A}$

- Aluminato férrico tetracálcico o Aluminato férrico, $4 \mathrm{CaO} \cdot \mathrm{Al}_{2} \mathrm{O}_{3}$. $\mathrm{Fe}_{2} \mathrm{O}_{3}$, abreviado: $\mathrm{C}_{4} \mathrm{AF}$

La concentración promedio de los componentes del material en bruto es, aproximadamente, del $65 \%$ en peso de $\mathrm{CaO}$, $20 \%$ de $\mathrm{SiO}_{2}, 6 \%$ de $\mathrm{Al}_{2} \mathrm{O}_{3}, 3 \%$ de $\mathrm{Fe}_{2} \mathrm{O}_{3}$, $2 \%$ de $\mathrm{MgO}, 2 \%$ de $\mathrm{SO}_{3}$, y de $1 \%$ a $2 \%$ de Alcalis.

Ellos están en disponibilidad como piedra caliza, arcilla, arena y material calcáreo (1-3).

\section{I.2 Modelo de reacciones en la cocción del clinker}

Las reacciones que intervienen en la formación del clinker (Fig. 1), pueden ser caracterizadas en los siguientes escalones de temperaturas (4):

- hasta una temperatura próxima a los $700^{\circ} \mathrm{C}$, se realiza la deshidratación del mineral arcilloso y unido a ello se observan un aumento de la actividad superficial y transformaciones de su estructura cristalina;

- entre los $700^{\circ} \mathrm{C}$ y $900^{\circ} \mathrm{C}$, se establece la desacidificación del $\mathrm{CaCO}_{3}$ con una simultánea formación de $\mathrm{Al}_{2} \mathrm{O}_{3}, \mathrm{Fe}_{2} \mathrm{O}_{3}$ y activación del $\mathrm{SiO}_{2}$ y $\mathrm{CaO}$;

- para una temperatura máxima de $1.200^{\circ} \mathrm{C}$ se observa que ya está concluída la formación de Belita $\left(\mathrm{C}_{2} \mathrm{~S}\right)$ a partir del $\mathrm{SiO}_{2}$ y $\mathrm{CaO}$ (cal libre) y además la transformación del aluminato férrico en $\mathrm{C}_{3} \mathrm{~A}$ y $\mathrm{C}_{4} \mathrm{AF}$;
Portland-Zementklinker, der mit ca. 95 Gew.\% den Hauptanteil des Zements darstellt, wird aus einem Rohstoffgemisch hergestellt, welches hauptächlich Calciumoxid $(\mathrm{CaO})$, Siliciumdioxid $\left(\mathrm{SiO}_{2}\right)$, Aluminiumoxid $\left(\mathrm{Al}_{2} \mathrm{O}_{3}\right)$ und Eisenoxid $\left(\mathrm{Fe}_{2} \mathrm{O}_{3}\right)$ enthält.

Durch Erhitzen dieses Gemischs bis zum Sintern bilden sich daraus neue Verbindungen, die sogenannten Klinkerphasen:

- Tricalciumsilicat oder Alit, $3 \mathrm{CaO} \mathrm{SiO}_{2}$, kurz: $\mathrm{C}_{3} \mathrm{~S}$.

- Dicalciumsilicat oder Belit, $2 \mathrm{CaO} \mathrm{SiO}_{2}$, kurz: $\mathrm{C}_{2} \mathrm{~S}$.

- Tricalciumaluminat oder Aluminat, $3 \mathrm{CaO} . \mathrm{Al}_{2} \mathrm{O}_{3}$, kurz: $\mathrm{C}_{3} \mathrm{~A}$.

- Tetracalciumaluminatferrit oder Aluminatferrit, $4 \mathrm{CaO} \cdot \mathrm{Al}_{2} \mathrm{O}_{3} \cdot \mathrm{Fe}_{2} \mathrm{O}_{3}$, kurz: $C_{4} A F$

Die durchschnittlichen Konzentrationen der Rohstoffkomponenten betragen atwa $65 \mathrm{Gew} . \% \mathrm{CaO}, 20 \% \mathrm{SiO}_{2}, 6 \% \mathrm{Al}_{2} \mathrm{O}_{3}$, $3 \% \mathrm{Fe}_{2} \mathrm{O}_{3}, 2 \% \mathrm{MgO}, 2 \% \mathrm{SO}_{3}$ und ca. 1-2\% Alkalien. Diese werden je nach Verfügbarkeit als Kalkstein, Ton und Quarzsand oder als Kalksteinmergel in genau berechneten Verhältnissen eingesetzt (1-3).

\section{I.2. Modell der Reaktionen beim Zementklinkerbrand}

Die Reaktionen der Klinkerbildung lassen sich modellhaft in folgenden Temperaturstufen charakterisieren [Abbildung 1, (4)]:

- bis ca. $700^{\circ} \mathrm{C}$ Entwässerung der Tonminerale, damit verbunden sind Gitterumwandlungen und eine Zunahme der Oberflächenaktivität;

- zwischen 700 und $900^{\circ} \mathrm{C}$ Calcination des $\mathrm{CaCO}_{3}$ und zugleich Bindung von $\mathrm{Al}_{2} \mathrm{O}_{3}$, $\mathrm{Fe}_{2} \mathrm{O}_{3}$ sowie aktiviertem $\mathrm{SiO}_{2}$ und $\mathrm{CaO}$;

- bis max. $1.200^{\circ} \mathrm{C}$ ist die Bildung von Belit $\left(\mathrm{C}_{2} \mathrm{~S}\right.$ ) aus $\mathrm{SiO}_{2}$ und $\mathrm{CaO}$ (Freikalk) abgeschlossen und es erfolgt eine Umwandlung der Calciumaluminatferrite zu $\mathrm{C}_{3} \mathrm{~A}$ und $\mathrm{C}_{4} \mathrm{AF}$; 
- entre los $1.250^{\circ} \mathrm{C}$ y $1.350^{\circ} \mathrm{C}$, se llevan a cabo las uniones de los enlaces alumino-férricos en el estado de fusión, comenzando la formación de Alita $\left(\mathrm{C}_{3} \mathrm{~S}\right)$ a partir de Belita y del $\mathrm{CaO}$ no combinado (libre);

- por encima de los $1.350^{\circ} \mathrm{C}$, ocurre una casi completa reacción de la cal libre hacia Alita, unido a una parcial descomposición de Belita;

- con el enfriamiento, se verifica la cristalización de la masa fundida en $\mathrm{C}_{3} \mathrm{~A}$ y $\mathrm{C}_{4} \mathrm{AF}$. Los silicatos $\mathrm{C}_{3} \mathrm{~S}$ y $\mathrm{C}_{2} \mathrm{~S}$ permanecen en forma y composición casi sin modificaciones.
- zwischen 1.250 und $1.350^{\circ} \mathrm{C}$ erfolgt der Übergang der aluminatischferritischen Verbindungen in den schmelzflüssigen Zustand und Beginn der Alitbildung $\left(\mathrm{C}_{3} \mathrm{~S}\right)$ aus Belit $\left(\mathrm{C}_{2} \mathrm{~S}\right)$ und ungebundenem $\mathrm{CaO}$ (Freikalk);

- oberhalb von $1.350^{\circ} \mathrm{C}$ erfolgt eine nahezu vollständige Reaktion des Freikalk zu Alit, verbunden mit einem weitgehenden Abbau des Belitanteils;

- beim abkühlen Kristallisation der Schmelze zu $C_{3} A$ und $C_{4} A F$. Die Silicate $C_{3} S$ und $C_{2} S$ bleiben dabei in Form und Zusammensetzung nahezu unverändert.

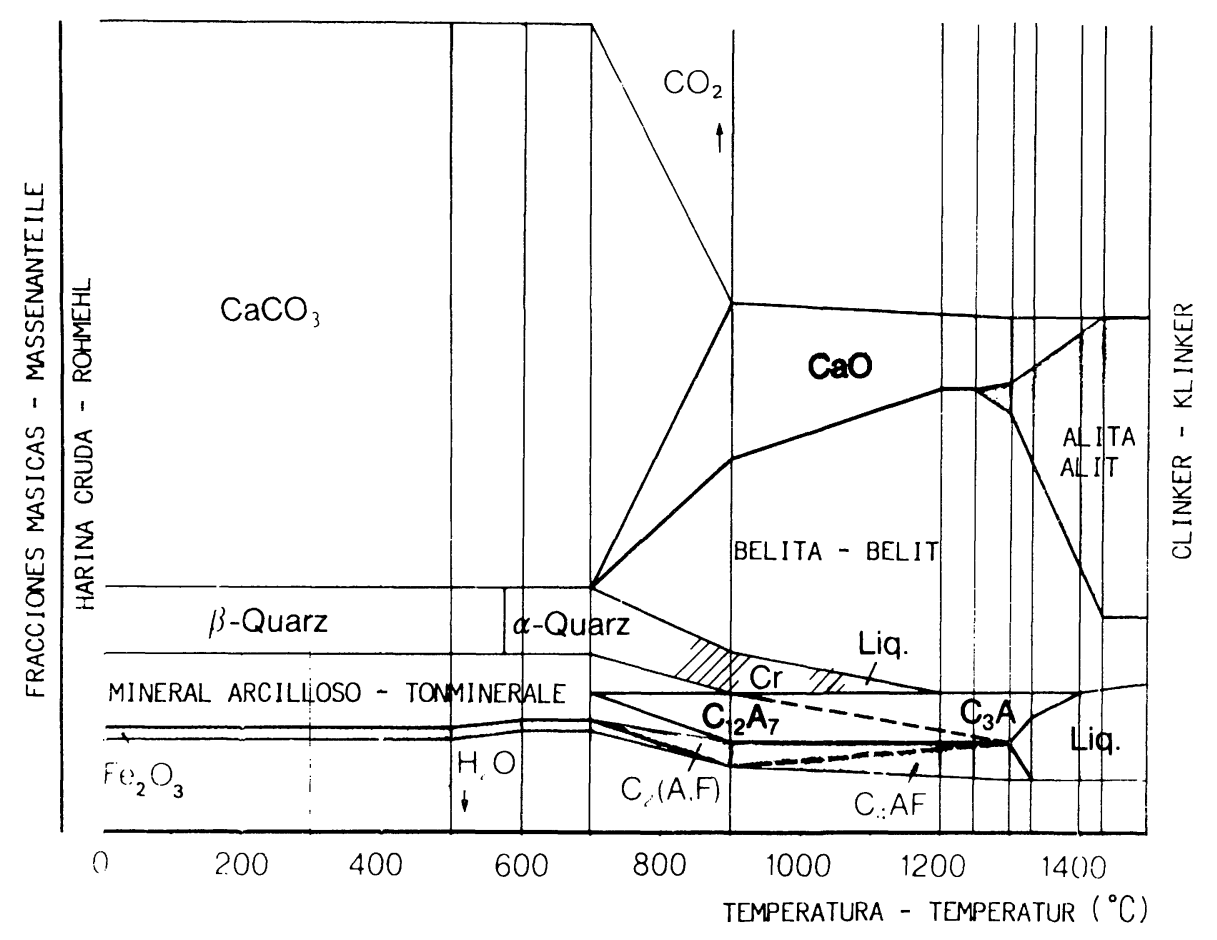

Fig. 1.-Representación esquemática de las reacciones en la formación del clínker.

Abbildung 1.-Schematische darstellung der klinkerbildungs-reactionen.

\section{I.3 Objeto del trabajo}

En los modernos procesos utilizados en la actualidad, se logra ya la desacidificación del material crudo antes de su ingreso al horno tubular rotativo mediante el uso de intercambiadores de calor y quemadores, siendo luego el material calcinado lentamente calentado hasta alcanzar su temperatura de sinterización. Así se conduce a una desactivación de la materia prima a través de la recristalización del $\mathrm{CaO}$ (libre) y parte de Belita.

\section{I.3. Aufgabenstellung}

Bei den heute verwendeten modernen Verfahren wird die Entsäuerung des Rohmeh/s durch Wärmetauscher und Zweitfeuerung bereits vor dem Eintritt in den Drehrohrofen erreicht. Danach wird das calcinierte Gut langsam bis zur Sintertemperatur aufgeheizt. Dies führt zu einer Desaktivierung der Rohstoffe durch Rekristallisation des Freikalkund Belitanteils. Der Klinkerbrand erfordert dann eine höhere Sintertemperatur oder eine längere Verweilzeit in der Sinterzone. Beides 
La cocción del clinker requiere pues una alta temperatura de sinterización o bien un prolongado tiempo en la zona de sinterización. Ambos procesos llevan a una clara ampliación de la estructura cristalina del clinker, resultando por consiguiente polvoriento y de difícil molienda.

Desde el punto de la tecnología de los procesos es de sumo interés que el tiempo de reacción necesario, para la formación del clinker, sea reducido a un mínimo. Por ello, el objetivo principal de la investigación se sitúa en la etapa de formación de las fases minerales del clinker en harinas crudas industriales, estudiándose en el presente trabajo el efecto de la utilización de altas cuotas de calentamiento.

\section{PROCEDIMIENTO EXPERIMENTAL}

Para la realización de la investigación fueron seleccionadas dos muestras industriales y utilizado un clinker modelo.

Como materia prima del clinker modelo se empleó carbonato de calcio, ácido silícico amorfo, y polvo de óxido de aluminio y hierro (III) de calidad proanálisis. Las muestras industriales fueron una mezcla parcialmente calcinada $\left(^{\star}\right)$ y una harina de cemento crudo $\left({ }^{\star \star}\right)$

El módulo de Cal Standard para la mezcla del clinker modelo fue ajustado a un valor de 98,3 ; el módulo de Alúmina a 2 y el módulo de Silicato a 3 . Estos parámetros están (en relaciones másicas) a continuación definidos:

\section{Cal Standard $=$}

$100 \mathrm{CaO}$

$2.8 \mathrm{SiO}_{2}+1.18 \mathrm{Al}_{2} \mathrm{O}_{3}+0.65 \mathrm{Fe}_{2} \mathrm{O}_{3}$

Módulo de Alúmina $=\frac{\mathrm{Al}_{2} \mathrm{O}_{3}}{\mathrm{Fe}_{2} \mathrm{O}_{3}}$

y Módulo de Silicato $=\frac{\mathrm{SiO}_{2}}{\mathrm{Al}_{2} \mathrm{O}_{3}+\mathrm{Fe}_{2} \mathrm{O}_{3}}$

Los reactantes fueron a través de suspensiones en éter de petróleo mezclados en primer lugar siendo a continuación secados y comprimidos en cantidades de

(*) Perteneciente a un yacimiento de Samawa/Irak

$\left.{ }^{\star \star}\right)$ Harina de cemento crudo procedente de Wössingen, estado de Baden-Württemberg/República Federal de Alemania. führt zu einer deutlich vergröberten Klinkerstruktur, und es resultieren staubige, schwer mahlbare Klinker.

Aus verfahrenstechnischer Sicht ist es daher von Interesse, die notwendige Reaktionszeit der Klinkermineralbildung beim Brand von Portland-Zementklinker auf das unbedingt notwendige Minimum zu reduzieren. In der vorliegenden Arbeit wird nun der Einflu $\beta$ hoher Autheizgeschwindigkeiten studiert, wobei der Schwerpunkt der Untersuchungen auf der Ausbildung der Klinkermineralphasen in technischen Rohmehlen liegt.

\section{EXPERIMENTELLES VORGEHEN}

Für die experimentellen Untersuchungen wurden zwei technische Rohmehle und ein Modellklinkermehl gewählt.

Ausgangsstoffe für das Modellklinkerrohmehl waren gefälltes Calciumcarbonat, amorphe Kieselsäure, Aluminiumoxid- und Eisen (III) oxid-Pulver in analysenreiner Qualität. Als technische Rohstoffe wurden ein teilcalciniertes Heißmehl $\left(^{*}\right)$ sowie ein Zementrohmehl (**) eingesetzt.

Bei der Modellklinkermischung wurde der Kalkstanderd auf 98.3, der Tonerdemodul auf 2 und der Silicatmodul auf einen Wert von 3 eingestellt. Hierbei sind die Kennzahlen (Massenverhältnisse) folgendermaßen definiert:

Kalkstandard $(\mathrm{KSt})=$

$$
=\frac{100 \mathrm{CaO}}{2.8 \mathrm{SiO}_{2}+1.18 \mathrm{Al}_{2} \mathrm{O}_{3}+0.65 \mathrm{Fe}_{2} \mathrm{O}_{3}}
$$

Tonerdemodul $(T M)=\frac{\mathrm{Al}_{2} \mathrm{O}_{3}}{\mathrm{Fe}_{2} \mathrm{O}_{3}}$

und der Silicatmodul $(\mathrm{SM})=\frac{\mathrm{SiO}_{2}}{\mathrm{Al}_{2} \mathrm{O}_{3}+\mathrm{Fe}_{2} \mathrm{O}_{3}}$

Die Reaktanden wurden durch Aufschlämmen in Petrolether zunächst inning vermischt, getrocknet und anschließend in Probenmengen von $750 \mathrm{mg}$ mit einer

(*) By-Pass-Ofenmehl einer Lagerstätte in Samawa/Irak. $\left.{ }^{\star \star}\right)$ Zementrohmehl aus Wössingen/Baden-Württemberg. 
$750 \mathrm{mg}$ en una prensa hidráulica, bajo una presión de $1.000 \mathrm{~kg} / \mathrm{cm}^{2}$. Como molde se utilizó una matriz de acero endurecido de $10 \mathrm{~mm}$ de diámetro, obteniéndose de esa manera unas tabletas cilíndricas de aproximadamente $5 \mathrm{~mm}$ de espesor.

Durante los rangos de calentamiento bajos y medios fue utilizado, y con excelentes resultados, un horno tubular instalado verticalmente, en tanto que las altas velocidades de $2.000,3.000$ y $5.000 \mathrm{~K} / \mathrm{min}$, pudieron ser alcanzadas solamente mediante la exposición directa de las muestras respectivas a la llama de un quemador.

El conjunto experimental para los ensayos a altas velocidades de calentamiento, realizados con la ayuda de un soplete circular de oxígeno/hidrógeno, se encuentra indicado en la Figura 2.
Hydraulikpresse unter einem Pre $\beta d r u c k$ von $1.000 \mathrm{~kg} / \mathrm{cm}^{2}$ verdichtet. Als Pre $\beta$ werkzeug diente eine gehärtete Stahlmatrize mit einem Stempeldurchmesser von $10 \mathrm{~mm}$. Die auf diese Weise erhaltenen Tabletten hatten eine Höhe von etwa $5 \mathrm{~mm}$. Während sich für niedere und mittlere Aufheizraten ein vertikal installierter Rohrofen bewährt hatte, ließen sich die hier erforderlichen Aufheizgeschwindigkeiten von $2.000,3.000$ und $5.000 \mathrm{~K} / \mathrm{min}$ nur durch eine direkte Erhitzung der Proben erreichen. Die Versuchsanordnung zur schnellen Aufheizung mit Hilfe eines Wasserstoff/SauerstoffGebläsebrenners ist in der Abbildung 2 gezeigt.
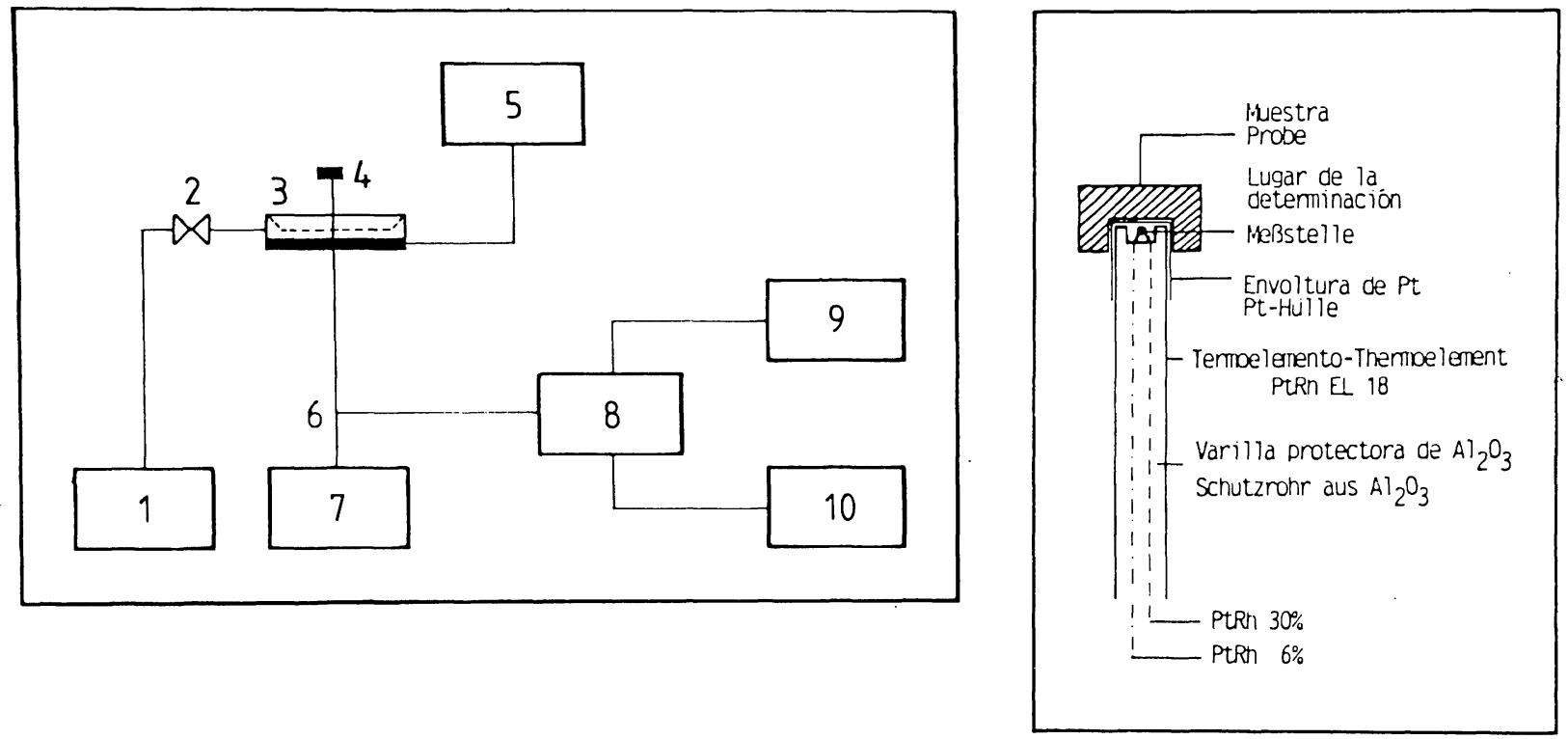

1. SUMINISTRO DE $\mathrm{H}_{2} \mathrm{YO}_{2}$

$\mathrm{H}_{2} / \mathrm{O}_{2}$ - VERSORGUNG

2. VALVULA DOSIFICADORA DOSIERVENTIL

3. QUEMADOR CIRCULAR DE MEZCLA GASEOSA RADIANTE HACIA ARRIBA

RINGFÖRMIGER, NACH OBEN ABSTRAHLENDER BRENNER IN MISCHGASAUSFÜHRUNG

4. MUESTRA A ENSAYAR PROBENANORDNUG

5. AGUA DE ENFRIAMIENTO WASSERKÜHLUNG
6. TERMOELEMENTO Pt Rh EL-18 Pt Rh EL-18 THERMOELEMENT

7. MOTOR TRANSPORTADOR DE LA MUESTRA MOTORGESTEUERTE PROBENEINFUHR

8. ELEMENTO DE REFERENCIA TERMICA (BANO DE HIELO, $0^{\circ} \mathrm{C}$ ) VERGLEICHSSTELLE (EISBAD, $\left.0^{\circ} \mathrm{C}\right)$

9. TERMOMETRO DIGITAL DIGITALES TEMPERATUR-ANZEIGEGERÄT

10. GRAFO-REGISTRADOR - $y, t$ $y, t-S C H R E I B E R$

Fig. 2.-Disposición esquemática del conjunto experimental de ensayos con un quemador de $\mathrm{H}_{2} / \mathrm{O}_{2}$.

Abbildung 2.-Schematischer aufbau der versuchsanordnung mit einem $\mathrm{H}_{2} / \mathrm{O}_{2}$-gebläsebrenner. 
Con la punta del termoelemento introducida en su parte media y mediante un servo motor de precisión, cada tableta a ensayar fue conducida cuidadosamente hacia la zona caliente de un quemador circular de radiación oblicua (de aproximadamente $45^{\circ}$ ). Luego de alcanzar la temperatura final de ensayo, se mantuvieron a la misma temperatura durante diferentes lapsos de tiempo, siendo finalmente enfriadas de manera rápida en nitrógeno líquido. El termoelemento fue conectado en paralelo con un termómetro digital y un grafo-registrador horizontal en donde sobre el papel fueron dibujadas previamente las curvas de calentamiento de $2.000,3.000$ y 5.000 $\mathrm{K} / \mathrm{min}$. Sobre ellas se condujo la pluma graficadora de manera precisa según los requerimientos de cada determinación, hasta el establecimiento de la temperatura deseada.

Una vez alcanzada la temperatura de $1.450^{\circ} \mathrm{C}$ a la velocidad prevista, las muestras permanecieron a dicha temperatura durante tiempos diferentes, siendo éstos hasta de 30 .

El progreso de la reacción fue seguido a través de la formación de óxido de calcio. Para ello, fue investigada analíticamente la cantidad no reaccionada o "cal libre", mediante extracciones de $\mathrm{CaO}$ o bien $\mathrm{Ca}(\mathrm{OH})_{2}$ con éster acetilacetacético y posteriores titulaciones con ácido Clorhídrico utilizando azul de bromofenol como indicador.

Ensayos de microscopía realizados en muestras inscrustadas y pulidas proporcionaron ulterior aclaración sobre el tamaño de los granos y el volumen de los poros.

\section{RESULTADOS Y DISCUSION}

\section{III.1 Resultados de las determinaciones de cal libre}

En las Figuras 3 y 4 se encuentran representadas las cantidades de $\mathrm{CaO}$ no combinado en función del tiempo de retención (t) de las muestras ensayadas.

Para alcanzar la temperatura de calcinación igual a $1.450^{\circ} \mathrm{C}$, es necesario un tiempo diferente de acuerdo a la velocidad de calentamiento. Así, las constituciones de las fases silicea-cálcicas establecidas ya por debajo de los $1.000^{\circ} \mathrm{C}$, ostentaron en las muestras más lentamente calentadas para un tiempo inicial $t_{0}=0 \mathrm{~min}$, valores muy pequeños de cal libre conforme a su naturaleza. La cuota de formación de cal, o sea la velocidad de decrecimiento de cal libre, disminuye con el aumento del tiempo. Causa
Die auf die Thermoelementspitze aufgesteckte Probe wurde mittels eines PräzisionsServomotors in die heiße Zone des schräg nach oben (unter einem Wilkel von $45^{\circ}$ ) abstrahlenden ringförmigen Brenners geschoben. Nach Erreichen der Solltemperatur wurden die Preßlinge unterschiedlich lange getempert und anschließend in flüssigem

Stickstoff schnell abgekühlt. Das

Thermoelement war mit einem digitalen Temperaturanzeigegerät und einem parallel dazu geschalteten $y, t$-Schreiber verbunden. Die auf dem Papier vorgezeichneten Thermospannungskurven für lineare Aufheizraten von $2.000,3.000$ und $5.000 \mathrm{~K} / \mathrm{min}$ wurden möglichst genau nachgefahren bis zum Erreichen der Solltemperatur.

Der Reaktionsfortschritt läßt sich anhand der Kalkbindung verfolgen. Dazu wurde der nicht gebundene oder auch "freie" Kalk naßchemisch bestimmt durch Extraktion von $\mathrm{CaO}$ oder $\mathrm{Ca}(\mathrm{OH})_{2}$ mit Acetessigsäureethylester und anschließender Titration der Lösung mit Salzsäure gegen Bromphenolblau als Indikator.

Lichtmikroskopische Untersuchungen an eingebetteten und polierten Proben sollten weiterhin Aufschlu $\beta$ darüber bringen, inwieweit sich Korngrö $\beta e n$ und Porenvolumina in den unterschiedlich behandelten Proben unterscheiden.

\section{ERGEBNISSE UND DISKUSSION}

\section{III.1. Ergebnisse der Freikalkanalysen}

In den Abbildungen 3 und 4 ist der Gehalt an nicht umgesetztem $\mathrm{CaO}$ gegen die isotherme Haltezeit ( $t$ ) aufgetragen. Bis zum Erreichen der Brenntemperatur von $1.450^{\circ} \mathrm{C}$ werden je nach Aufheizrate unterschiedliche Aufheizzeiten benötigt. Da die Bildung von Calciumsilicatphasen aber bereits unterhaib von $1.000^{\circ} \mathrm{C}$ einsetzt, weisen langsamer aufgeheizte Proben bei $t_{0}=0$ min naturgemä $\beta$ geringere Freikalkwerte auf. Die Kalkbindungsraten, d.h. die Geschwindigkeiten der Freikalkabnahme, werden mit zunehmender Zeit kleiner. Ursache hierfür sind die immer länger werdenden Diffusionswege bei der geschwindigkeitsbestimmenden Reaktion $\mathrm{CaO}+\mathrm{C}_{2} \mathrm{~S} \rightarrow \mathrm{C}_{3} \mathrm{~S}$. Man erkennt einen positiven Einflu $\beta$ höherer 
de ello, es la utilización de los caminos siempre largos de difusión de la reacción determinante $\mathrm{CaO}+\mathrm{C}_{2} \mathrm{~S} \rightarrow \mathrm{C}_{3} \mathrm{~S}$. Se reconoce una positiva influencia de las más altas velocidades de calentamiento, solamente poco después de alcanzar la temperatura final de ensayo. Con el crecimiento del período de calcinación, los valores se aproximan unos a
Aufheizgeschwindigkeiten lediglich kurz nach Erreichen der Solltemperatur. Mit zunehmender Brenndauer nähern die Werte sich einander an (Abbildung 4). Als Vergleich dazu sind die Ergebnisse an den gleichen Rohmehlen und derselben Brenntemperatur für niedere und mittlere Aufheizgeschwindigkeiten gegenübergestellt (Abbildung 3). Auch hier

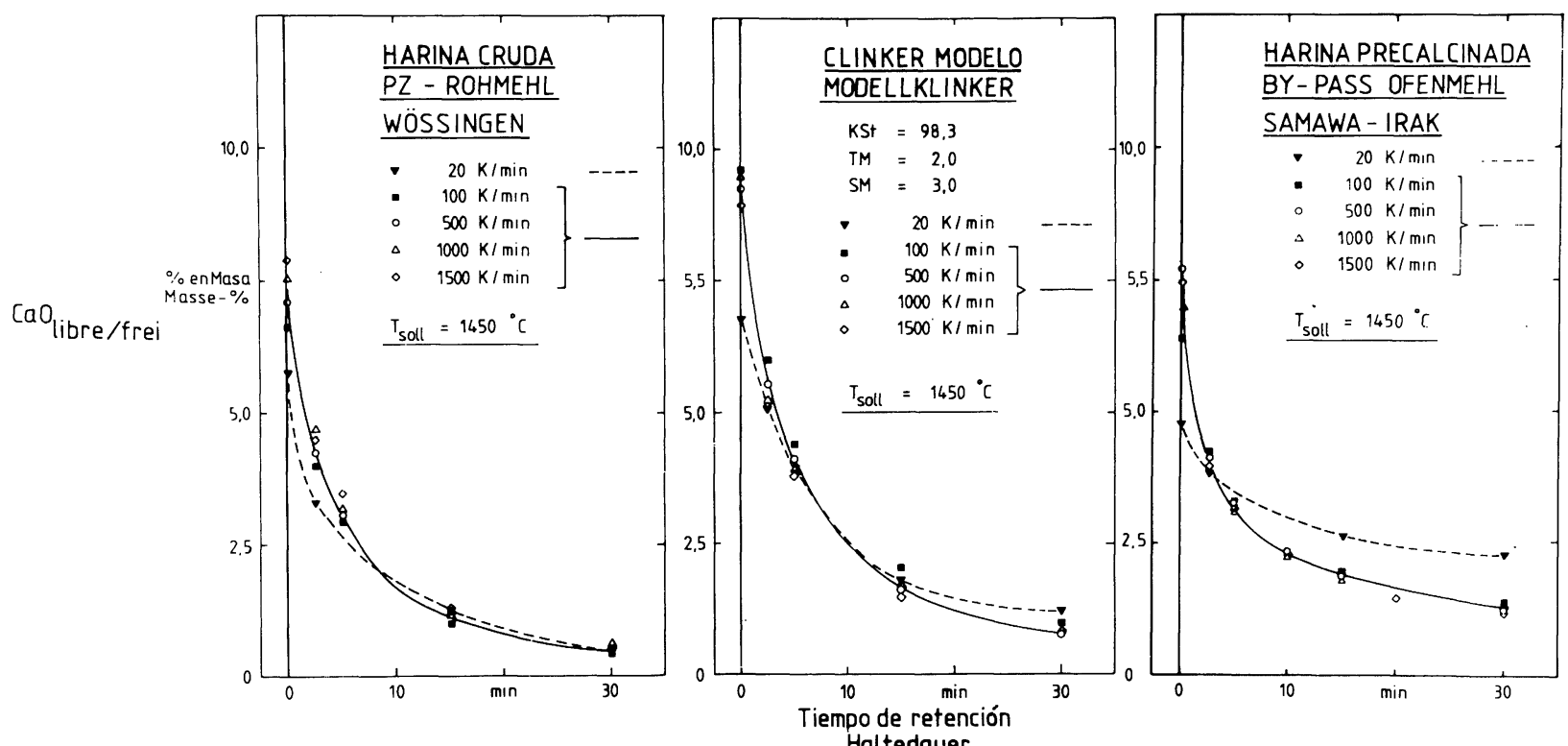

Fig. 3.- Influencia de las bajas y medias velocidades del calentamiento sobre el decrecimiento de cal libre en el clínker modelo y en las harinas crudas industriales para una temperatura de calcinado de $1.450^{\circ} \mathrm{C}$.

Abbildung 3.-Einflu $\beta$ niederer und mittlerer aufheizraten auf die freikalkabnahme eines modellrohmeh/s und technischer zementrohmehle bei einer brenntemperatur von $1.450^{\circ} \mathrm{C}$.

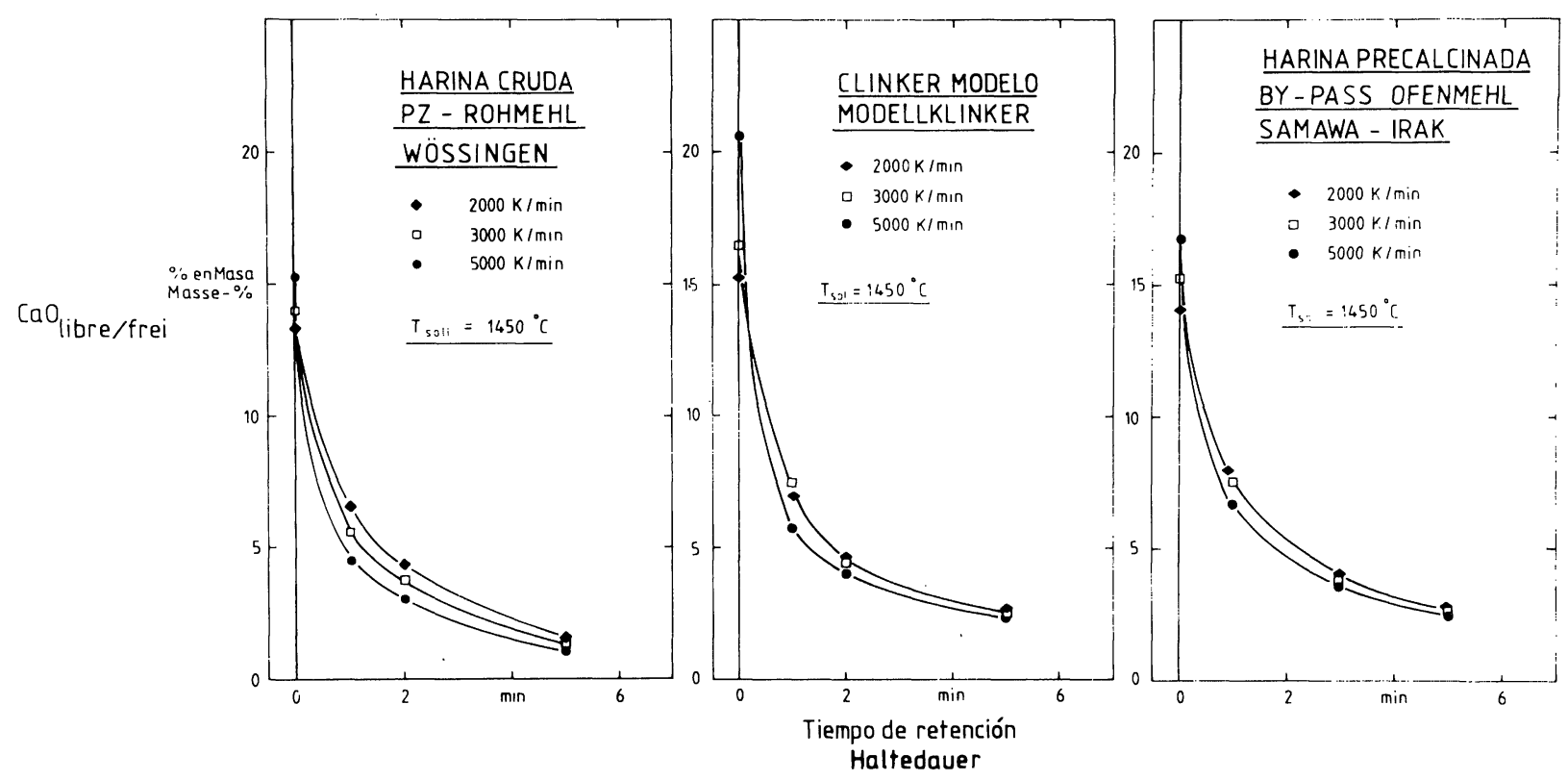

Fig. 4.- Influencia de las altas velocidades de calentamiento sobre el decrecimiento de cal libre en el clínker modelo y en las muestras industriales para una temperatura de $1.450^{\circ} \mathrm{C}$.

Abbildung 4.-Einfluß schneller aufheizraten auf die freikalkabnahme eines modellrohmehls und technischer zementrohmehle bei einer brenntemperatur von $1.450^{\circ} \mathrm{C}$. 
TABLA 1 TABELLE 1

Valores del período de calcinación a punto $\left(\mathrm{CaO}_{\mathrm{LIBRE}} \leq 2 \%\right.$ en masa) obtenidos gráficamente para el clínker modelo y muestras industriales, en ensayos a distintos calentamientos

Graphisch ermittelte garbranddauern $\left(\mathrm{CaO}_{F R E I} \leq 2\right.$ masse-\%) von modellklinker- und technischen klinkerproben nach verschieden schneller aufheizung

\begin{tabular}{|c|c|c|}
\hline $\begin{array}{l}\text { TIPO DE MUESTRA } \\
\text { ART DER PROBE }\end{array}$ & $\begin{array}{l}\text { VELOCIDAD DE CALENTAMIENTO } \\
\text { AUFHEIZGESCHWINDIGKEIT } \\
(\mathrm{K} / \mathrm{min})\end{array}$ & $\begin{array}{c}\text { PERIOOO DE CALCINACION } \\
\text { A PUNTO } \\
\text { GARBRANDDAUER } \\
(\mathrm{min})\end{array}$ \\
\hline $\begin{array}{l}\text { CLINKER MODELO } \\
\text { MODELL-KLINKER }\end{array}$ & $\begin{array}{r}20 \\
100 \\
500 \\
1000 \\
1500\end{array}$ & $\begin{array}{l}13.5 \\
12.0 \\
12.0 \\
12.0 \\
12.0\end{array}$ \\
\hline $\begin{array}{c}\text { Ensayo a la llama } \\
\text { Brennerversuche }\end{array}$ & $\begin{array}{l}2000 \\
3000 \\
5000\end{array}$ & $\begin{array}{l}6.6 \\
6.4 \\
6.0\end{array}$ \\
\hline $\begin{array}{l}\text { HARINA PRECALCINADA } \\
\text { BY-PASS OFENMEHL } \\
\text { (SAMAWA IRAK) }\end{array}$ & $\begin{array}{r}20 \\
100 \\
500 \\
1000 \\
1500\end{array}$ & $\begin{array}{l}38.5 \\
13.0 \\
13.0 \\
13.0 \\
13.0\end{array}$ \\
\hline $\begin{array}{l}\text { Ensayo a la llama } \\
\text { Brennerversuche }\end{array}$ & $\begin{array}{l}2000 \\
3000 \\
5000\end{array}$ & $\begin{array}{l}6.9 \\
6.5 \\
6.2\end{array}$ \\
\hline $\begin{array}{c}\text { HARINA CRUDA DE } \\
\text { CEMENTO } \\
\text { PZ-ROHMEHL } \\
\text { (wÖSSINGEN) }\end{array}$ & $\begin{array}{r}20 \\
100 \\
500 \\
1000 \\
1500 \\
\end{array}$ & $\begin{array}{l}9.0 \\
8.5 \\
8.5 \\
8.5 \\
8.5\end{array}$ \\
\hline $\begin{array}{l}\text { Ensayo a la llama } \\
\text { Brennerversuche }\end{array}$ & $\begin{array}{l}2000 \\
3000 \\
5000\end{array}$ & $\begin{array}{l}4.2 \\
3.8 \\
3.2\end{array}$ \\
\hline
\end{tabular}


otros como se aprecia en la Figura 4. Como comparación, están los resultados para bajas y medias velocidades de calentamiento de iguales materiales e iguales temperaturas confrontados (Figura 3). También aquí se reconocen claras diferencias, sobre todo poco después de alcanzar la temperatura final.

En el clinker modelo y en especial en el material parcialmente calcinado del horno de Samawa, existen también para los más largos períodos de calcinación diferentes cuotas de formación de cal. Una negativa influencia de los rápidos calentamientos sobre los subsiguientes calcinados, no fue establecido mediante pruebas experimentales.

Como aproximación y a modo de relacionar con la práctica, son designadas a aquellas muestras cuyo contenido de cal libre es $\leq 2 \%$ en masa, muestras con reacción completa o "cocidas a punto". De aquí, el tiempo de calcinación necesario para cumplir esa condición es denominado Tiempo de Reacción Completa o "Período de Calcinación a Punto".

Los valores averiguados gráficamente de los diagramas observados, se presentan resumidos en la Tabla 1.

La aceleración de la velocidad de calcinado actúa más fuertemente sobre el material precalcinado de Samawa. Durante el aumento de la velocidad de calentamiento de $20 \mathrm{~K} / \mathrm{min}$ a $100 \mathrm{~K} / \mathrm{min}$, es el Período de Calcinación a Punto acortado a algo menos de la mitad. Los continuos aumentos de la velocidad de calentamiento no han brindado, por el contrario, una importante mejora, siendo esto último válido también tanto para el clinker modelo como para las harinas crudas industriales.

En la Tabla 1 están especificados, además, los valores del Periodo de Calcinación a Punto para los ensayos de extremo calentamiento. Sin embargo, se debe considerar que los valores absolutos de $\mathrm{CaO}$ libre de las muestras ensayadas, directamente a la llama, sólo pueden ser comparados en forma relativa con los valores obtenidos en el horno tubular. La razón para ello es el sobrecalentamiento local de las muestras en el calcinado directo. Esta limitación concierne sólo a la contribución del Período de Calcinación a Punto y no en cambio al cualitativo transcurso del de crecimiento de cal libre.

\section{III.2 Ensayos de microscopia}

Las primeras diferencias de las fases del sind deutliche Unterschiede vor allem kurz nach Erreichen der Solltemperatur erkennbar. Bei dem Modellklinkerrohmehl und besonders bei dem teilcalcinierten Ofenmehl aus Samawa sind auch nach längerer Brenndauer unterschiedliche Kalkbindungsraten vorhanden. Ein negativer Einflu $\beta$ der schnellen Aufheizung auf den nachfolgeden Klinkergarbrand wurde bei keiner Versuchsserie festgestellt. In Annäherung an die Verhältnisse in der Praxis werden die Klinkerproben, deren Freikalkgehalt $\leqq 2$ Masse-\% beträgt, als gergebrannt bezeichnet. Die hierfür notwendige Brennzeit ist als "Garbranddauer" des betrachteten Rohmeh/s definiert. Die aus den Schaubildern graphisch ermittelten Garbranddauern sind in der Tabelle 1 zusammengestellt.

Die Beschleunigung der

Brenngeschwindigkeit wirkt sich am stärksten aus beim teilcalcinierten Rohmehl aus Samawa. Durch Steigerung der Aufheizrate von $20 \mathrm{~K} / \mathrm{min}$ auf $100 \mathrm{~K} / \mathrm{min}$ wird die Garbranddauern auf mehr als die Hälfte verkürzt. Die weitere Erhöhung der Aufheizgeschwindigkeit bringt dagegen keine wesentliche Verbesserung. Dies gilt auch für die Modellklinker und technischen Rohmehlproben.

Daneben sind in Tabelle 1 die für extrem hohe Aufheizraten ermittelten Garbranddauern aufgeführt. Dabei mu $\beta$ man bedenken, da $\beta$ die Absolutwerte für freies $\mathrm{CaO}$ aus den Gasbrennerversuchen nur bedingt mit den Werten der Ofenversuche verglichen werden können. Der Grund dafür ist die örtliche Überhitzung der Proben bei direkter Beheizung. Diese Einschränkung betrifft aber nur den Betrag der Garbranddauern, nicht jedoch den qualitativen Verlauf der Freikalkabnahme; d.h. mit Raten von 2.000 bis $5.000 \mathrm{~K} / \mathrm{min}$ aufgeheizte Rohmehle unterscheiden sich analog dem Aufheizbereich von 100 bis $1.500 \mathrm{~K}$ min nur unwesentlich in ihren Garbranddauern.

\section{III.2. Mikroskopische Untersuchungen}

\section{Erste Unterscheidungen von Klinkerphasen in}


clinker pudieron ser encontradas en probetas no grabadas, mediante observaciones microscópicas de luz reflejada (5). Estos preparados pulidos, pero sin tratar, fueron sin embargo inapropiados para un detallado análisis estructural. Buenos resultados, concerniente al impregnado y grabado de la estructura de las fases Alita, Belita, Aluminato Aluminato férrico y de la cal libre, suministró una solución alcohólica al $1 \%$ de ácido Nítrico, la que por pocos segundos se dejó reaccionar sobre la altamente pulida superficie de la probeta a ensayar (6). De esta manera, las muestras pretratadas fueron nuevamente observadas, procediéndose a las mediciones con la ayuda de un ocular calibrado.

El tamaño de los cristales formados admitió una dependencia con el tratamiento térmico realizado sobre las harinas crudas. No ha sido considerada la influencia de la velocidad de enfriamiento, debido a que luego del correspondiente tiempo de retención, todas las muestras fueron rápidamente enfriadas en nitrógeno líquido. Los tamaños promedios de los cristales medidos en $(\mu \mathrm{m})$ se encuentran representados en función al tiempo de retención para diferentes ensayos de rápidos calentamientos, en las Figuras 5, 6 y 7.

El tamaño promedio de los cristales de Alita (Fig. 5) aumenta con el crecimiento de la velocidad de calentamiento, observándose que los mismos, inicialmente para $t_{0}=0$ min, presentan los menores valores en las muestras calentadas más rápidamente.

Totalmente inversa es la relación del tamaño del cristal de Belita con el aumento de la velocidad de calentamiento. Aquí, las muestras calentadas más lentamente proporcionaron los más grandes cristales. Se observa además una disminución de tamaño en las muestras con mayores tiempos de retenciones.

La recristalización de $\mathrm{C}_{2} \mathrm{~S}$ a causa de la reacción $\mathrm{CaO}+\mathrm{C}_{2} \mathrm{~S} \rightarrow \mathrm{C}_{3} \mathrm{~S}$ está correlacionada con la recristalización de $\mathrm{CaO}$, ya que también las muestras expuestas a menores velocidades de calentamiento presentaron grandes cristales de $\mathrm{CaO}$.

Los cristales de $\mathrm{CaO}$ libre son microscópicos y de difícil observación, por lo que fueron evaluados a través de sus aglomeraciones naturales denominadas "nidos de $\mathrm{CaO}$ libre". Se señala una clara disminución de la formación de los conglomerados con el crecimiento de la velocidad de calentamiento.

Cualitativamente, un curso semejante muestran los diagramas del clinker modelo (Fig. 6) y del precalcinado material de Samawa (Fig. 7). ungeätzten Proben konnten anhand der Intensität des reflektierten Lichts getroffen werden [siehe auch (5)]. Ungeätzte Anschliffe eigneten sich jedoch nicht für detaillierte Phasen, -Gefüge- und Strukturentersuchungen. Gute Ergebnisse bezüglich der Anlauf- und Strukturätzung von Alit, Belit, Ferrit, Aluminat und Freikalk lieferte eine $1 \%$-ige alkoholische Salpetersäurelösung, die man mehrere Sekunden auf die hochpolierte Probenoberfläche einwirken lä $\beta t$ (6). die so vorbehandelten Klinkerschliffe wurden mit Hilfe eines Strichokulars vermessen. Die Größe der gebildeten Klinkerkristalle läßt einen Rückschlu $\beta$ auf die thermische Vorbehandlung der Rohmehle zu. $\mathrm{Da}$ alle Proben nach entsprechender isothermer Haltezeit sehr schnell in flüssigem Stickstoff abgekühlt wurden, braucht die Abkühlgeschwindigkeit als beeinflussende Größe nicht berücksichtigt werden. Die durchschnittliche Kristallit-Korngröße in $\mu \mathrm{m}$ ist in der Abbildung 5 gegen die isotherme Haltezeit nach verschieden schneller Aufheizung aufgetragen. Die durchschnittliche Alit-Korngröße nimmt mit steigender Aufheizgeschwindigkeit $z u$, wobei die Primärkeimgröße bei $t_{0}=0$ min für die höchste Aufheizrate den kleinsten Wert besitzt. Gerade umgekehrt ist die Abhängigkeit der Belit-Korngröße von der Höhe der Autheizgeschwindigkeit. Hier zeigen die langsam aufgeheizten Proben nach entsprechender Haltezeit größere Belit-Kristalle. $\mathrm{Da}$ die $\mathrm{C}_{2} \mathrm{~S}$-Rekristallisation aufgrund der Reaktion $\mathrm{CaO}+\mathrm{C}_{2} \mathrm{~S} \rightarrow \mathrm{C}_{3} \mathrm{~S}$ mit der CaO-Rekristallisation korrelierbar ist, sind bei langsam aufgeheizten Proben auch größere CaO-Kristalle zu erwarten. Einzelne Freikalk-Kristalle sind mikroskopisch nur schwer erfassbar, weshalb nach größeren CaO-Kristallansammlungen, sogenannten "Freikalk-Nestern" ausgewertet wurde. Es zeigte sich eine deutliche Abnahme der Nesterbildung bei Erhöhung der Auíheizgeschwindigkeit. Qualitativ gleiche Verläufe zeigen die Diagramme für Modellklinkerproben (Abbildung 6) und das teilcalcalcinierte Ofenmehl aus Samawa (Abbildung 7). Die durchschnittliche Alit Korngröße nimmt mit steigender Aufheizrate $z u$, während die Belit-Kristalle bei langsamer Aufheizung stärker rekristallisieren. In verschiedenen Arbeiten wurde übereinstimmend festgestellt, daß schnell gebrannte Klinker besser mahlbar sind; die zur Klinkermahlung notwendige Energie und der Verschlei $\beta$ der Mahlaggregate wird verringert (7-9). Die beim Aufheizen erzeugte Oberflächen und Feh/stellenaktivität führt zu einer gleichmäßigen Granulation und zur Ausbildung kleinerer Calciumsilicat-Kristalle. Trotz des geringeren Mahlaufwands weisen schnell gebrannte Klinker bei gieichem Kalkstandard höhere Frühfestigkeiten auf ais langsam 
También en ellos el tamaño promedio de los granos de Alita va en aumento con el crecimiento de la velocidad de calentamiento, mientras que los cristales de Belita gebrannte Klinker $(10,11)$. Neben den in den Diagrammen 5-7 dargestellten mittleren Klinkerkristall-Korngrößen wurden mit steigender Aufheizgeschwindigkeit

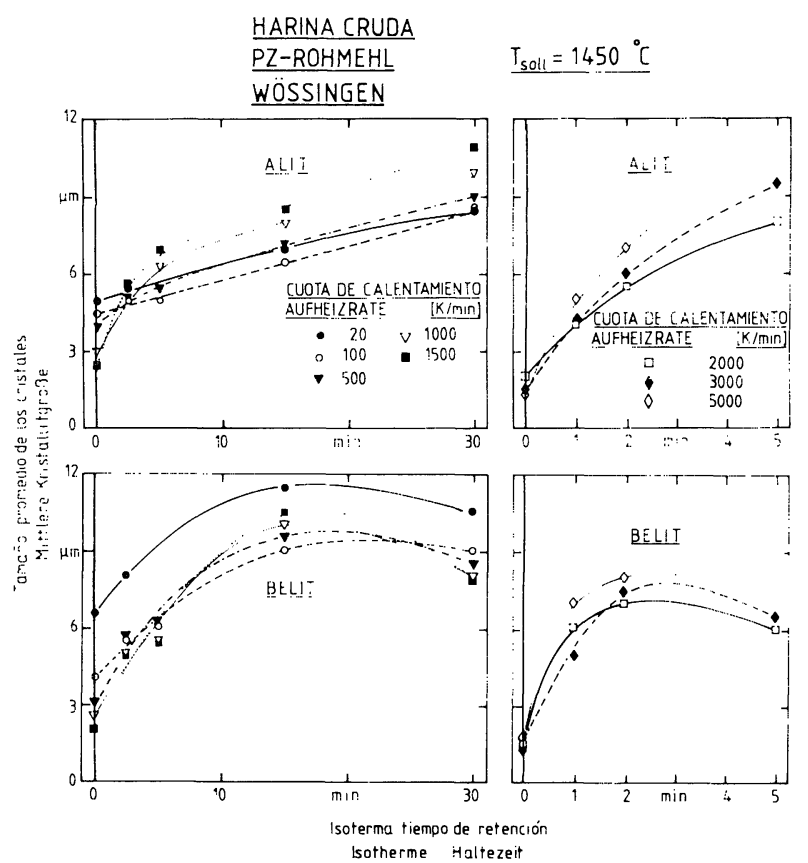

Fig. 5-Tamaño promedio de los cristales de alita y belita en función del tiempo de retención luego de distintos calentamientos rápidos.

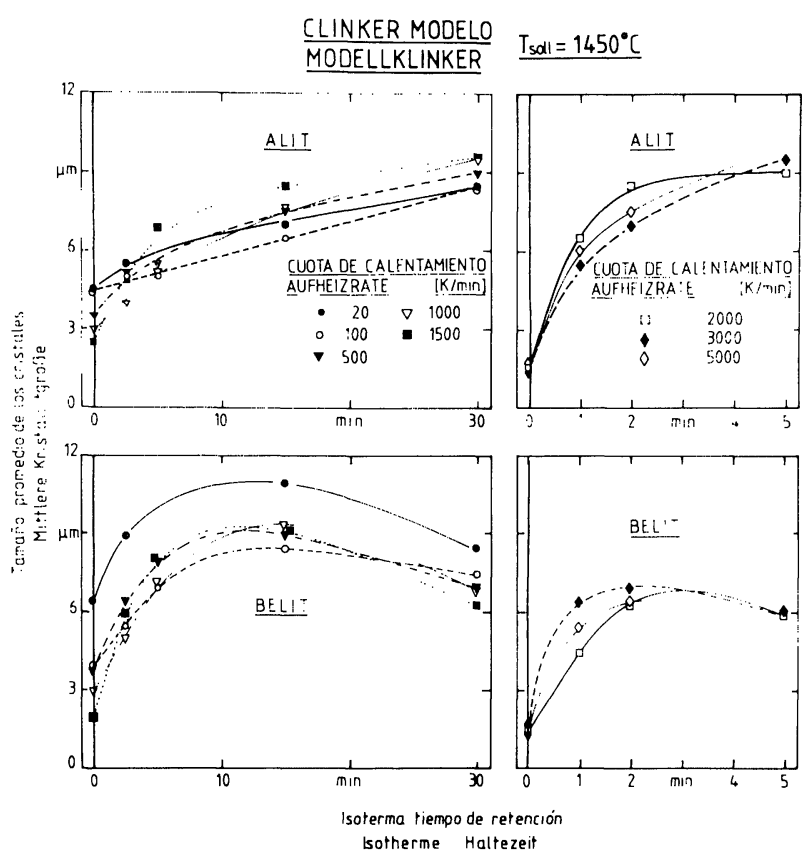

Fig. 6.-Tamaño promedio de los cristales de alita y belita del clínker modelo luego de distintos calentamientos rápidos.

Abbildung 6.-Durchschnittliche alit- und belit- korngrö $\beta e$ von modellklinker nach verschieden schneller autheizung.
Abbildung 5.-Mittlere alit- und belit- Kristallitgröße in abhängigkeit von der isothermen haltedauer nach verschieden schneller aufheizung.

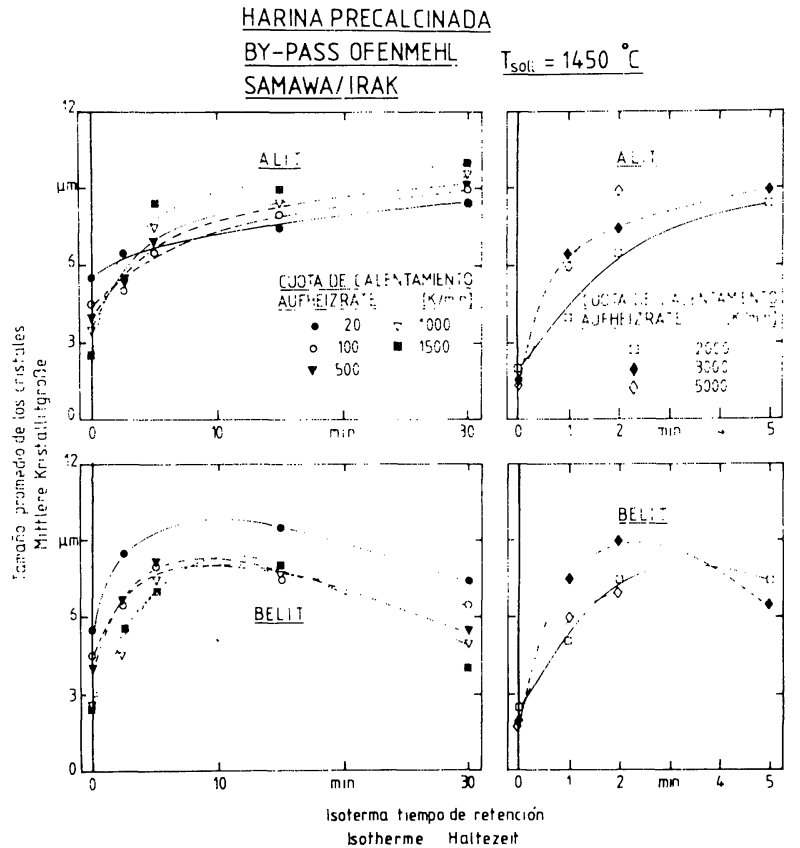

Fig. 7.- Tamaño promedio de los cristales de alita y belita del parcialmente calcinado material de Samawa/Irak, luego de distintos calentamientos rápidos.

Abbildung 7.-Mittlere kristall-korngrößen von alit- und belit-phasen nach unterschiedlich schneller autheizung; teilcalciniertes ofenmehl aus Samawa/lrak. 
recristalizan más fuertemente en los procesos más lentos.

En diferentes trabajos y en forma coincidente se ha establecido que los clínkeres obtenidos por procesos de calcinación rápida son los mejores para la posterior operación de molienda, ya que la cantidad de energía necesaria y el desgaste ocasionado en los elementos del molino, se ven fuertemente disminuidos (7-9).

Este proceso de calcinación produjo una defectuosa actividad superficial, conduciendo a una uniforme granulación y formación de cristales mas pequeños de silicatos. Pese a los relativamente bajos costos de molienda, ha quedado de manifiesto que los clínkeres rapidamente calcinados con iguales módulos de Cal Standard presentaron mas altas resistencias iniciales que los clínkeres calentados a bajas velocidades (10-11).

En las Figuras 5, 6 y 7 se observan además en los diagramas correspondientes a los ensayos de extremo calentamiento, que un ascenso de la velocidad produjo también un aumento de los microcristales. Por consiguiente, los materiales de este trabajo procesados con estas velocidades de calentamiento deberían pasar, por lo menos, a obtener similar calidad de producto final y poseer mejores propiedades de molienda.

\section{CONCLUSIONES}

Los resultados obtenidos en los estudios realizados sobre el clinker modelo y las muestras industriales, han demostrado que el parámetro "Velocidad de Calentamiento" puede acelerar, para una predeterminada temperatura de calcinado, las reacciones de formación del clinker. En tanto una influencia negativa de las altas velocidades no fue establecido mediante la serie de ensayos.

El tamaño de las partículas primarias y también el de las secundarias es disminuido, sobre todo el de Belita y cal libre, influyendo en la reactividad de las fases de transición.

La significativa presencia de la fase de microcristales, luego de las altas cuotas de calentamiento, conducen a una supuesta disminución de los gastos de energía en función de menores costos de molienda. Estudios de otros autores demostraron que un clínker rápidamente calcinado ostenta también un más alto endurecimiento inicial. zunehmende Anteile mikrokristalliner Phasen festgestellt. Daher sollten auch die hier mit hohen Aufheizraten gebrannten Klinker bessere Mahleigenschaften bei wenigstens gleichbleibender Klinkerqualität besitzen.

\section{SCHLUSSFOLGERUNGEN}

Die bisherigen Ergebnisse der Untersuchungen an Modellklinkerproben und technischen Rohmehlen haben gezeigt, da $\beta$ der Parameter "Autheizgeschwindigkeit" die Verklinkerungsreaktionen bei der vorgegebenen Brenntemperatur beschleunigen kann. Ein negativer Einflu $\beta$ der schnellen Aufheizung in Form einer schlechteren Brennbarkeit wurde bei keiner Versuchsserie festgestellt

Die Primärteilchengröße wird durch schnelles Autheizen verringert und auch die Sekundärkorngrößen der für die Reaktivität verantwortlichen Übergangsphasen -vor allem Belit und Freikalk - werden kleiner.

Der hohe Anteil mikrokristalliner Phasen nach vorheriger schneller Aufheizung führt vermutlich zu einem geringeren Energieaufwand bei der kostenintensiven Klinkermahlung. Wie Untersuchungen anderer Autoren zeigten, weisen schnell gebrannte Klinker auch höhere Anfangsfestigkeiten auf. 
Junto a un aumento de los rendimientos de tiempo y espacio, a través de una contracción del tiempo de calentamiento entre la desacidificación y la sinterización, se podría también obtener, operando con altas cuotas de calentamiento, productos de buena calidad, conforme a lo postulado por Hedvall (12-13) que un aumento de la reactividad de la materia prima por activación térmica, puede como consecuencia permitir una mayor superficie activa a través de la producción de muchos y pequeños cristales primarios.
Neben einer Erhöhung der

Raum-Zeit-Ausbeute durch Verkürzung der Aufheizzeit zwischen dem Entsäuerungs - und dem Sinterbereich können durch den Klinker- Schnellbrand auch noch Qualitätsverbesserungen erreicht werden.

Die von Hedvall $(12,13)$ postulierte erhöhte Reaktionsfähigkeit von Rohstoffen nach vorheriger thermischer Aktivierung kann somit als Folge eines vergrößerten Angebots an reaktiver Oberfläche durch Erzeugung vieler, kleiner Primärkristalle verstanden werden.

\section{v. LITERATURA - LITERATUR}

(1) H. KÜHL.: "Zement-Chemie". Band II, VEB-Verlag für Technik, Berlin, 1958.

(2) Verein Deutscher Zementwerke, "Zement-Taschenbuch", 48. Ausgabe, Bau-Verlag, Wiesbaden-Berlin, 1984.

(3) F. W. LOCHER: "Hundert Jahre Forschung über Zementchemie in Deutschland", S. 57-73, in Verfahrenstechnik der Zementherstellung, Bau-Verlag, Wiesbaden-Berlín, 1979.

(4) A. WOLTER: "Einflu $\beta$ des Ofensystems auf die Klinkereigenschaften", ZKG, 38, (1985), S. 612-614.

(5) E. J. OSEN: "Untersuchungen zur Kinetik der Phasenbildung im System Calciumoxid-Siliciumdioxid", Diplomarbeit am Institut für Chemische Technik der Universität Karlsruhe (TH), 1983.

(6) Verein Deutscher Zementwerke, "Mikroskopie des Zementklinkers Bilderatlas", Beton-Verlag, Düsseldorf, 1965.

(7) J. M. BUTT, V. M. KOLBASOV, G. A. MELNIEKIJ: "Zur Wechselbeziehung zwischen der Porenstruktur und Mahlbarkeit von Klinkern aus verschiedenen Brenn- und Abkühlungsverfahren", ZKG, 27, (1974), S. 27-32.

(8) G. R. GOULA: "Effect of clinker composition on grindability", Cem. Concr. Res., 9, (1979), S. 209-218.

(9) B. SCHEUBEL, H. J. BALZER: "Mikroskopische Untersuchungen an Zement-klinkern mit Hilfe der Linearanalyse zur Bestimmung der Klinkermahlbarkeit". ZKG, 36, (1983), S. 624-627.

(10) P. FRÜHAUF, S. E. IBRAHIM, U. LUDWIG: "Einfluß der Aufheizgeschwindingkeit und der Kühlung auf die Bildung und Eigenschaften des Zement-klinkers", TIZ, 103, (1979), S. 594-602.

(11) J. GRZYMEK: "Die Bedeutung der äußeren Gestalt der Alit-Kristalle für die Erzeugung von frühhochfesten Portlandzementen", Silikattechnik, 6, (1955), S. 296-303.

(12) J. A. HEDVALL: "Einführung in die Festkörperchemie", Verlag Vieweg \& Sohn, Braunschweig, 1952.

(13) J. A. HEDVALL: "Sinterung und Festkörperaktivität", Ber. Dt. Keram. Ges., 30, (1953), S. 8-13. 